\title{
Les sciences sociales et les deux relativismes
}

\section{Raymond Boudon}

\section{OpenEdition}

\section{Journals}

Édition électronique

URL : http://journals.openedition.org/ress/531

DOI : $10.4000 /$ ress.531

ISSN : 1663-4446

\section{Éditeur}

Librairie Droz

\section{Édition imprimée}

Date de publication : 1 septembre 2003

Pagination : 17-33

ISBN : 2-600-00878-0

ISSN : 0048-8046

Référence électronique

Raymond Boudon, "Les sciences sociales et les deux relativismes », Revue européenne des sciences sociales [En ligne], XLI-126 | 2003, mis en ligne le 30 novembre 2009, consulté le 19 avril 2019. URL http://journals.openedition.org/ress/531; DOI : 10.4000/ress.531 


\title{
Raymond BOUDON
}

\section{LES SCIENCES SOCIALES ET LES DEUX RELATIVISMES}

\author{
LE RELATIVISME, \\ THÈSE FONDAMENTALE DES SCIENCES SOCIALES \\ CONTEMPORAINES
}

Dans les trente dernières années les sciences sociales ont beaucoup contribué à rendre le relativisme crédible sous ses deux formes du relativisme cognitif et du relativisme culturel. On peut même dire que le relativisme représente l'une des thèses fondamentales de la sociologie et de l'anthropologie contemporaines, ainsi qu'une dimension essentielle de leur influence et de leur contribution à la vision «postmoderniste» du monde. Faut-il croire avec les relativistes cognitifs qu'il n'y a pas de connaissance objective, mais seulement des interprétations du réel? Faut-il croire avec les relativistes culturels que les normes et les valeurs sont le produit de l'«arbitraire culturel»?

Le relativisme pose d'intéressants problèmes de sociologie de la connaissance et d'épistémologie: pourquoi ces deux formes du relativisme ont-elles gagné en influence dans les dernières décennies? Pourquoi, alors que le relativisme philosophique n'a jamais été qu'une école de pensée parmi d'autres, le relativisme cognitif et le relativisme culturel se présentent-ils comme des thèses confirmées par les sciences sociales modernes? Quelles sont les données et les arguments sur lesquels les deux formes du relativisme s'appuient? Quelle est leur valeur? D'où provient leur influence? Les deux formes du relativisme ont-elles la même diffusion? Sont-elles promises au même avenir?

Telles sont quelques-unes des questions que je souhaite aborder ici. J'envisagerai successivement le cas du relativisme cognitif, puis celui du relativisme culturel.

\section{LE RELATIVISME COGNITIF}

\section{Le rôle de Kuhn}

Le relativisme cognitif s'installe dans les sciences sociales sous l'influence de Kuhn (1962). La Structure des révolutions scientifiques insiste sur l'idée que l'histoire des sciences est moins linéaire que ne le disent les manuels d'histoire et de philosophie des sciences. Les discussions scientifiques font apparaître des échanges d'arguments qui ne sont pas toujours « rationnels» (au sens que la philosophie des sciences prête à cet adjectif), souligne-t-il. L'adhésion du scientifique 
à une théorie se fait sur la base de critères qui peuvent être par exemple esthétiques ou philosophiques, voire politiques tout autant que rationnels.

De telles idées paraissent parfaitement acceptables. Il paraît même curieux, avec le recul, que La Structure des révolutions scientifiques ait été perçue comme un ouvrage révolutionnaire, et qu'il ait mis en marche la «nouvelle sociologie des sciences » qu'évoque Bunge (1999): un mouvement de pensée qui devait produire des visions de plus en plus radicalement relativistes de la connaissance scientifique, et, en fin de compte, dénier aux sciences toute capacité d'aboutir à une vision «objective» des phénomènes naturels; des visions faisant des théories scientifiques des constructions dont l'objectivité ne serait pas davantage garantie que celle des représentations du monde proposées par les mythologies; qui seraient des «contes de fée», ainsi que l'a assuré Feyerabend (1975).

Pourquoi ce relativisme s'est-il imposé? Pourquoi a-t-il pris des formes extrêmes? Le point de départ de ce processus provient de ce que Kuhn s'appuie sur des observations monographiques convaincantes. Il est vrai que, lorsqu'on analyse les controverses scientifiques dans le détail, on constate que le choix du scientifique pour telle ou telle théorie s'opère pour partie en fonction de critères «irrationnels ». Il est vrai d'autre part que cette vue contredit les thèses de la philosophie des sciences qui étaient courantes au moment où Kuhn écrit, celles que Popper avait, par exemple, développées sous l'étiquette du «rationalisme critique». Il est vrai enfin que les manuels scolaires véhiculaient et véhiculent toujours une représentation «rationnelle» de l'histoire des sciences.

On comprend donc que Kuhn, bien qu'il ait énoncé des propositions qui peuvent facilement passer pour banales, ait donné l'impression de proposer une révolution dans la conception courante des sciences. La révolution qu'il avait déclenchée se greffait sur un noyau solide: le fait historiquement attesté que les processus de sélection des théories scientifiques sont moins rationnels que ne le disent l'histoire classique des sciences et la philosophie des sciences.

\section{Du Cercle de Vienne à Popper}

Deuxième facteur de la pénétration de Kuhn et de la radicalisation de ses idées par ses émules: les échecs de la philosophie des sciences. Kuhn apparut comme d'autant plus révolutionnaire et d'autant plus solide que ses vues étaient contradictoires avec celles de la philosophie des sciences, mais aussi parce que la philosophie des sciences apparaît au moment où il les expose comme étant bloquées dans une impasse.

On peut dater l'histoire de la philosophie moderne des sciences des travaux du Cercle de Vienne. Le Cercle de Vienne se pose notamment une question: comment cerner la distinction entre science et non-science? Où passe la ligne de démarcation entre science et non-science? Quels critères permettent-ils de déterminer si une théorie est scientifique ou non? La réponse la plus connue, celle de Carnap à une étape de sa pensée, consiste à affirmer qu'une théorie scientifique est, en principe du moins, une théorie qui, une fois entièrement déployée, apparaît comme se composant d'un ensemble de propositions protocolaires: de propositions enregistrant des données irrécusables tirées de l'observation des faits.

Cette réponse a été critiquée. Popper a proposé, comme on sait, une autre ligne de démarcation entre science et non-science: le critère de la réfutabilité. Une 
théorie scientifique est, selon lui, une théorie qui peut être réfutée à partir des données fournies par l'observation: c'est une théorie qui se soumet au verdict du réel. Ce critère de démarcation a été longtemps considéré comme acceptable. Il est vrai que la théorie du phlogistique peut être plus facilement réfutée ou, comme on dit maladroitement (mais à la suite de Popper lui-même), «falsifiée » que la monadologie de Leibniz. Selon Popper, il n'est pas de théorie qui puisse être qualifiée de vraie, mais seulement des théories vraisemblables: celles qui, au vu de tous les tests auxquels on a imaginé de les soumettre, n'ont pas été rejetées par la «réalité». Mais, en dépit de sa modestie, la théorie popperienne ne capte la distinction science / non-science que de manière très imparfaite. Pour plusieurs raisons.

- En premier lieu: parce qu'il existe des propositions «falsifiables » qu'on aurait peine à tenir pour scientifiques, comme: «le train part à 8 h. 47 ».

- En deuxième lieu, parce qu'on considère normalement comme scientifiques toutes sortes de théories qui ne peuvent par construction être falsifiées, comme ces théories économiques qui introduisent la clause du «toutes choses égales d'ailleurs », sans proposer de moyens pour déterminer si la clause en question est satisfaite.

- En troisième lieu, parce que certaines théories ne portent pas directement sur le réel, mais proposent plutôt des «cadres » permettant de construire des théories sur le réel; par suite, elles ne sauraient être tenues à proprement parler pour réfutables. C'est le cas de la théorie darwinienne de l'évolution. Popper lui-même a reconnu qu'il était difficile de ne pas la considérer comme scientifique et en même temps qu'elle n'était pas directement falsifiable.

- En quatrième lieu, parce que, comme l'indique la thèse dite de Duhem-Quine, il n'est pas plus facile de se convaincre qu'une théorie est fausse que de se convaincre qu'elle est vraie. Quant une théorie qui a fait ses preuves apparaît comme incompatible avec certains faits, nous dit cette thèse, on ne peut savoir d'avance si une modification mineure de la théorie ne parviendra pas à la réconcilier avec les faits en question. Qu'une théorie soit contredite par certains faits n'est donc nullement un motif suffisant de rejet. L'histoire des sciences paraît effectivement donner raison à Duhem et à Quine. Un scientifique ne renonce pas à une théorie qui a fait ses preuves parce qu'il constate qu'elle est incompatible avec un fait nouveau. Il cherche plutôt à l'amender.

En résumé, la conjoncture intellectuelle des années 1960 se caractérise, d'une part par l'attention accordée à la théorie de Kuhn selon laquelle la sélection des idées scientifiques est moins rationnelle que ne le disent l'histoire et la philosophie des sciences et, d'autre part, par la perte de crédibilité de toutes les théories selon lesquelles il existerait des critères généraux de démarcation entre science et non-science.

\section{L'utilisation abusive du principe du tiers exclu}

Joue alors un mécanisme cognitif dont l'action est facilement repérable en maintes circonstances: l'utilisation abusive du principe du tiers exclu. Elle 
consiste en particulier à ériger des termes contraires en termes contradictoires. Exemple: si un objet n'est pas blanc, c'est qu'il est noir.

C'est bien une utilisation abusive du principe du tiers exclu qu'on observe ici: ou bien la sélection des idées scientifiques est rationnelle ou elle ne l'est pas. $O u$ bien l'on peut établir le ou les critère(s) de démarcation entre science et nonscience, ou bien on ne le peut pas. S'il n'est pas possible de déterminer les critères de démarcation entre science et non-science, c'est qu'il n'y en a pas et que la distinction entre science et non-science est une illusion. D'où l'on conclut qu'une théorie scientifique ne saurait prétendre à être plus objective qu'une interprétation mythique des phénomènes naturels. Le mécanisme cognitif de l'abus du principe du tiers exclu conduit de Kuhn à Feyerabend.

\section{Les «écluses» de Scheler et la distinction paretienne entre le «vrai» et l'«utile»}

Il faut encore, pour compléter cette analyse, évoquer un autre facteur responsable de l'audience du relativisme cognitif. Max Scheler a indiqué que, selon les conjonctures, une idée pouvait ou non franchir des «écluses» virtuelles: toutes choses égales d'ailleurs, une idée a plus de chances d'être acceptée si elle est congruente avec l'air du temps ou avec tel ou tel type d'intérêts collectifs. Pareto a indiqué dans la même veine que, pour comprendre l'accueil fait à une théorie, il faut examiner, non seulement si elle est «vraie», si elle est crédible, mais si elle est «utile». Il veut dire par-là que certaines idées sont endossées, non seulement parce qu'elles paraissent crédibles, mais parce qu'elles correspondent à des intérêts cognitifs, idéologiques ou matériels; à des intérêts de caste, de classe, de groupe, etc.

C'est ce qui s'est passé dans le cas qui nous occupe. La critique de la science qui se déploie à partir de Kuhn correspond à une conjoncture où est dénoncée aux Etats-Unis la collusion entre politiques, scientifiques et militaires. Cette collusion se fait, ajoute-t-on, sur le dos du peuple et au grand dam de la démocratie. C'est l'époque de la dénonciation de la technostructure militaro-industrielle. Les «écluses » de Scheler s'ouvrirent alors tout naturellement aux théories ayant pour effet de détrôner la science, de la faire descendre du piédestal sur lequel historiens traditionnels et philosophes des sciences l'avaient placée. Cet effet n'avait pas été recherché par Kuhn. Il n'avait peut-être même pas été voulu par les sociologues relativistes des sciences les plus radicaux. Mais les théories «constructivistes » de la science étaient «utiles» au sens de Pareto: elles étaient congruentes avec des intérêts politiques et idéologiques.

Les théories «constructivistes » de la science étaient non seulement «utiles », de surcroît elles paraissaient «vraies ». La recherche des critères de démarcation entre science et non-science paraissait introuvable. Les processus de sélection des idées scientifiques apparaissaient effectivement comme beaucoup moins rationnels que ne le voulaient l'histoire et la philosophie des sciences. C'est pourquoi le relativisme cognitif s'imposa. Pour un temps.

Tout simplifié qu'il soit, je crois que ce scénario rend compte de la réalité, qu'il explique bien le succès du mouvement d'idées qui va de Kuhn aux constructivistes. 
Du point de vue de la sociologie de la connaissance, il a, de plus, l'intérêt d'identifier un processus typique. Il décrit l'installation de bien d'autres idées douteuses. On le verra en examinant le cas du relativisme culturel. Ce processus se caractérise par l'existence d'un ou plusieurs noyaux solides; par un mécanisme d'hyperbolisation de ces noyaux à partir de procédés familiers à la connaissance ordinaire, comme l'utilisation abusive du principe du tiers exclu; par une congruence entre les théories hyperboliques ainsi obtenues et des intérêts politiques et/ou idéologiques.

\section{Le déclin du «constructivisme»}

Pourquoi le même constructivisme est-il aujourd'hui sur le déclin? Durkheim (1979[1910], p. 624) a dit, dans Les Formes élémentaires de la vie religieuse, qu'il arrive souvent que l'on croie dans un premier temps à une idée parce qu'elle est collective; mais, ajoute-t-il aussitôt, pour que la croyance collective en question se maintienne, il faut qu'elle soit perçue comme fondée: «nous lui demandons ses titres avant de lui accorder notre créance».

Le cas du relativisme cognitif contemporain illustre l'importance de cette idée.

On a cru dans un premier temps au «constructivisme» parce que beaucoup y croyaient, notamment parmi les «spécialistes». Il fut un temps, naguère encore, avant que Sokal ne lance son canular et que Sokal et Bricmont (1997) ne publient leur livre, où il était difficile de faire passer dans une revue de sociologie des sciences, ou même dans une revue de vulgarisation scientifique, en France surtout, un article qui ne fasse pas allégeance de manière plus ou moins discrète, au constructivisme et au relativisme. Pourquoi cette ère paraît-elle révolue? Par les raisons qu'indique Durkheim dans le passage que je viens d'évoquer: parce que le constructivisme allait bien au-delà des noyaux solides que représentaient d'une part les idées de Kuhn et d'autre part le constat d'échec dressé à l'encontre de Popper s'agissant de la détermination des critères de démarcation entre science et non-science.

En un mot: le constructivisme a commencé à s'étioler parce qu'on a pris conscience du fait que l'utilisation de ces noyaux présentait un caractère hyperbolique. Le public diffus qu'il avait conquis a alors commencé à s'évaporer. La croyance collective que représentait le relativisme cognitif n'a pas été confirmée: apparaissant comme non fondée, son public diffus a cessé de lui accorder sa «créance», pour parler comme Durkheim. Les médiateurs ont cessé de le soutenir.

Pour résumer très brièvement les raisons de cette disqualification, on peut relever les deux arguments suivants.

1) Comme l'indique justement Kuhn, la sélection des idées scientifiques fait apparaître sur le court terme l'action de facteurs irrationnels. Cela n'exclut pas que, sur le long terme, cette sélection ne soit rationnelle. Lavoisier a objectivement raison contre Priestley même si, comme l'a montré Kuhn, la discussion de la théorie du phlogistique de Priestley fait apparaître que les prises de position des hommes de science impliqués dans le débat furent souvent inspirées par des motivations de caractère «irrationnel».

C'est seulement si l'on néglige la distinction entre le court et le long terme que l'on peut appliquer le principe du tiers exclu et déclarer que, la discussion 
entre savants n'étant pas rationnelle, la sélection des idées scientifiques doit être tenue pour irrationnelle. Dès lors que l'on introduit une distinction entre le court et le long terme, la question de savoir si les discussions entre savants sont rationnelles ou non est disqualifiée.

2) Il en va de même s'agissant des conséquences qui ont été tirées de l'échec des efforts faits par la philosophie des sciences pour déterminer les critères de démarcation entre science et non-science. On ne les a pas trouvées. Mais, comme Kant l'a indiqué, ce n'est pas parce qu'il n'existe pas de critères généraux du vrai que le vrai n'existe pas. Il n'existe pas de critères généraux de la vérité, mais on peut, dans bien des cas, trancher en faveur d'une théorie contre une autre. C'est ce que nous enseigne l'histoire des sciences. On peut accepter la théorie du baromètre de Torricelli-Pascal de préférence aux théories d'inspiration aristotélicienne parce qu'elle rend mieux compte des phénomènes observés et qu'elle n'introduit pas l'idée que la nature aurait horreur du vide. Plus généralement, on évoque normalement toutes sortes de distinctions qui paraissent évidentes et auxquelles on serait bien en peine de faire correspondre des critères précis. Ainsi, il est évident que la distinction entre la musique classique et la musique populaire n'est pas illusoire, mais aussi qu'on aurait du mal à indiquer les critères généraux qui correspondent à cette distinction.

Appliquée à notre problème, cette proposition nous dit que l'impossibilité de déterminer des critères généraux de distinction entre science et non-science n'exclut pas que cette distinction existe bel et bien; on détermine le plus souvent facilement, dans des cas particuliers, si une théorie est scientifique ou si elle ne l'est pas; mais il n'existe pas de critères généraux permettant de distinguer le monde de la science de celui de la non-science. L'on en conclut que l'échec à identifier des critères de démarcation n'implique ni qu'il n'y ait pas de démarcation entre science et non-science, ni qu'il faille abonder dans le sens du constructivisme et du relativisme cognitif.

Le constructivisme cognitif s'est donc bien effectivement étiolé pour les raisons qu'a indiquées Durkheim: nous voyons maintenant qu'il est de caractère hyperbolique. Nous sommes désormais en mesure de «déconstruire » la croyance collective qui régna naguère à ce propos en sociologie des sciences. Les nouveaux Protagoras sont désormais disqualifiés. Le relativisme cognitif paraît en déroute. Sokal et Bricmont ont contribué à précipiter ce processus.

\section{LE RELATIVISME CULTUREL}

Le relativisme culturel doit être distingué du relativisme cognitif. Le second est surtout le fait d'une corporation étroite, celle des sociologues des sciences, dont l'influence ne s'étend guère au-delà d'un public restreint.

Le relativisme culturel est beaucoup plus largement diffusé que le relativisme cognitif. D'autre part, alors que le relativisme cognitif est en déclin, le relativisme culturel apparaît comme beaucoup plus tenace.

Si je juge utile de les rapprocher, c'est qu'ils ont un trait commun. Sur ce point, je me sépare de Rod Aya (2001). Dans les deux cas, les croyances collectives 
correspondantes se sont installées sous l'effet du processus général que j'ai identifié dans le cas du relativisme cognitif. Ses composantes sont: l'existence d'un ou de plusieurs noyaux durs; l'apparition d'un effet d'hyperbolisation à partir de ces noyaux durs par mise en œuvre de mécanismes cognitifs comme l'utilisation abusive du principe du tiers exclu; un effet de renforcement dû à l' «utilité » du relativisme culturel: à sa congruence avec des intérêts individuels ou collectifs, matériels ou symboliques.

Je commencerai par les noyaux durs. Le relativisme culturel s'appuie sur trois noyaux durs qu'on peut associer à trois figures emblématiques: Montaigne, Hume et Max Weber. Elles ont beaucoup inspiré les analystes contemporains.

\section{Montaigne}

L'anthropologue américain Clifford Geertz (1984) est l'auteur d'un article influent, intitulé Anti anti-relativism, qui s'appuie explicitement sur un chapitre célèbre des Essais de Montaigne: l'Apologie de Raymond Sebond. "Quelle vérité que ces montagnes bornent, qui est mensonge au monde qui se tient au-delà?». Montaigne écrit à l'époque des guerres de religions. Son objectif dans ce chapitre était sans doute avant tout de caractère politique: en suggérant qu'il n'y a pas de vérité en matière de normes, il voulait inciter les Catholiques et les Protestants à cesser de se massacrer. Mais Geertz ne s'intéresse pas à ces données circonstancielles.

L'attention de Geertz a sans doute été attirée par un passage comme le suivant. Il indique que, selon Montaigne, la culture est pour l'homme une seconde nature: «Il est crédible qu'il y a des lois naturelles [...]; mais en nous elles sont perdues [...] Une nation regarde un sujet par un visage [...]; l'autre par un autre». Et Montaigne d'illustrer son propos, selon son habitude, par une série d'évocations concrètes. Par exemple: «Il n'est rien si horrible à imaginer que de manger son père. Les peuples qui avaient anciennement cette coutume, la prenaient toutefois pour témoignage de piété et de bonne affection, cherchant par-là à donner à leurs progéniteurs la plus digne et honorable sépulture, logeant en eux-mêmes et comme en leurs moelles les corps de leurs pères et leurs reliques. [...] Il est aisé à considérer quelle cruauté et abomination c'eût été, à des hommes abreuvés et imbus de cette superstition, de jeter la dépouille des parents à la corruption de la terre et nourriture des bêtes et des vers » (Les Essais, II, XII, Paris, Garnier, 1948, p. 289).

Selon Geertz, Montaigne doit être pris au pied de la lettre. Il aurait par avance énoncé une vérité essentielle, consolidée par l'anthropologie moderne: la vérité selon laquelle, en matière normative, il n'y aurait pas de vérité, mais seulement des coutumes variables d'une société à l'autre. Toute distinction entre coutumes d'un côté, normes et valeurs de l'autre serait illusoire. Les raisons que les sujets sociaux perçoivent comme fondant leurs croyances ne sauraient être que des justifications ou des rationalisations, mais non les causes de ces croyances. Ces causes seraient à rechercher du côté de forces culturelles émises par toute société, i.e. du système de règles en vigueur. Les comportements et les croyances seraient au premier chef des effets de la socialisation.

Ce noyau dur est facilement repérable chez beaucoup d'anthropologues. On l'observe chez les auteurs d'hier: chez Lévy-Bruhl (1960), Granet (1990), 
Whorf (1969) ou Needham (1972); ou d'aujourd'hui: chez Geertz ou Shweder; on l'observe aussi chez des politologues comme Goldhagen (1997) ou Huntington (1996). Les uns et les autres sont convaincus que le monde est constitué de cultures discontinues correspondant à des systèmes de normes et de valeurs propres à chacune; et que ces cultures émettent des forces expliquant les comportements et les croyances des individus.

Dans cette veine, un article de Shweder (2000) évoque le témoignage d'une anthropologue africaine élevée aux États-Unis, qui, une fois retournée dans son pays, la Sierra-Leone, s'est soumise à la pratique de l'excision. La plupart des femmes Kono, déclare-t-elle dans une communication présentée devant la société américaine d'anthropologie, tirent de l'excision un sentiment de pouvoir accru; exactement comme les hommes Kono de la circoncision. Shweder en conclut qu'il faut reconnaître comme un fait incontestable l'existence de forces culturelles qui conduisent le sujet social à percevoir telle règle de façon positive ou négative; elles expliquent qu'une Sierra-Leonaise élevée aux Etats-Unis ait perçu la pratique de l'excision comme positive au point de se faire exciser. Shweder pousse même le bouchon plus loin: il verse à l'appui de son analyse une étude due à une anthropologue de Harvard, selon laquelle les effets négatifs de l'excision sur un plan médical auraient été grandement exagérés. Shweder suggère d'interpréter ce résultat comme indiquant que le jugement négatif porté sur cette pratique par l'observateur occidental résulte de préjugés: qu'il est l'effet de forces à l'œuvre dans les sociétés occidentales.

Montaigne fournit donc au relativisme culturel un premier noyau dur: l'infinie diversité des règles prescriptives, des normes et des valeurs exclurait que celles-ci puissent être fondées sur autre chose que sur des «conventions culturelles» arbitraires, dont l'origine est le plus souvent inconnue, mais qui s'imposent à l'individu au cours de la socialisation.

\section{Hume}

Le second noyau dur a une influence plus diffuse. Il s'agit du célèbre théorème de Hume, selon lequel aucun raisonnement à l'indicatif ne peut se terminer par une conclusion à l'impératif: un théorème inébranlable. On en a tiré l'idée qu'un gouffre séparerait le positif du normatif, le descriptif du prescriptif, le factuel de l'axiologique; que l'adhésion à des jugements normatifs ne saurait provenir de ce qu'ils seraient objectivement fondés, puisqu'ils ne sauraient l'être par principe, l'impératif ne pouvant être tiré de l'indicatif.

Cette idée a eu, me semble-t-il, une influence considérable. C'est peut-être parce qu'il a pris Hume au pied de la lettre que le philosophe Ayer a forgé l'hypothèse que les jugements normatifs seraient des commandements voilés. Ils exprimeraient le désir que quelque chose se fasse. Ils ont l'apparence d'affirmations («il est bien de faire $X »)$; mais ces affirmations seraient l'expression indirecte d'un désir. Le sentiment de certitude ou d'évidence associé par le sujet à un jugement normatif serait une illusion. Une telle théorie ne peut, je crois, qu'éveiller un sentiment d'arbitraire, sauf si l'on est convaincu que les jugements normatifs sont par essence indémontrables.

Je m'attarderai davantage sur les anthropologues. Nombre d'entre eux peuvent, comme ceux que j'ai déjà évoqués, être placés sous le pavillon du culturalisme, si 
l'on accepte de donner à cette notion un sens large et non le sens étroit qui l'associe aux travaux de Kardiner et de son école. Ces anthropologues «culturalistes» au sens large sont ceux qui analysent le sentiment de certitude qui accompagne les croyances normatives endossées par les sujets qu'ils observent, sinon comme des illusions, du moins comme des effets de la socialisation; comme des "vérités » passivement acceptées, dont le sujet lui-même ne voit pas le sens et auxquelles il attribue normalement un sens qu'elles n'ont pas. Le sujet social étant ainsi décrit comme entièrement aveugle sur lui-même, ce serait donc au sociologue ou à l'anthropologue qu'il appartiendrait de restituer les causes véritables de ses croyances.

Les anthropologues qui adoptent le noyau dur de Montaigne ont peut-être été aussi plus ou moins indirectement impressionnés par le noyau dur de Hume. Car Hume donne pour ainsi dire un fondement théorique à l'observation empirique de Montaigne. Si les règles sont indéfiniment variables d'une culture à l'autre, cela ne provient-il pas de ce que l'impératif ne peut résulter de l'indicatif ? S'il est vrai que les faits eux-mêmes n'imposent aucune norme, n'en découle-t-il pas que les normes sont nécessairement «conventionnelles»?

Maintes études anthropologiques se donnent en effet pour objectif de montrer, non seulement que d'innombrables normes varient d'une culture à l'autre, mais qu'il en va de même de celles que l'observateur occidental perçoit facilement comme ayant une vocation à l'universalité, comme les critères de l'équité. Ainsi, une étude impressionnante par le plan d'observation ambitieux sur lequel elle repose (Henrich et al., 2001) constate que les réponses à ce classique de la psychologie sociale qu'est le jeu de l'ultimatum varient selon les cultures, suggérant que le sens de l'équité lui-même n'a rien d'universel.

Non seulement une majorité d'anthropologues, mais de nombreux sociologues greffent leurs travaux sur les deux noyaux durs de Montaigne et de Hume. La différence entre les deux corporations est que, là où les anthropologues s'intéressent à des sociétés globales, les sociologues s'intéressent plutôt à des segments sociaux: correspondant aux professions, aux classes sociales, etc. Comme ils observent un de ces segments à l'autre des variations dans les systèmes de normes et de valeurs auxquels obéissent les sujets sociaux, leur conclusion est identique à celle des anthropologues de tendance culturaliste: les normes et les valeurs correspondant aux divers segments sont perçues sur le mode de l'évidence par les sujets eux-mêmes; mais, comme ces évidences sont différentes d'un segment à l'autre, ce sentiment d'évidence ne saurait être qu'une illusion. Cette démarche est illustrée en Angleterre par Mary Douglas et Ney (1998) ou, en France, par les cités de Boltanski et Thévenot (1991), qui rebaptisent sous ce vocable nouveau les souscultures d'hier.

Il me semble que l'adhésion à cette perspective «culturaliste» n'est possible que pour qui est indéfectiblement convaincu que les croyances axiologiques (les croyances concernant les normes et les valeurs) ne sauraient être objectivement fondées. Or Hume est celui qui, en apparence, a donné le fondement le plus clair à cette thèse.

\section{Max Weber}

Max Weber a fourni aux sciences sociales un troisième noyau dur. De nombreux commentateurs le présentent comme un hyper-relativiste sur la base 
notamment de deux de ses métaphores: le «polythéisme des valeurs» et la «guerre des dieux». Ces métaphores évoquent facilement l'idée que les sociétés sont habitées par des valeurs et des normes incompatibles entre elles. Elles suggèrent que la vie des sociétés serait faite, moins de lutte des classes, que de conflits de valeurs.

Ces interprétations courantes constituent un troisième noyau dur: le plus grand des sociologues (avec Durkheim) aurait insisté sur le fait que les valeurs et les normes relèvent de l' «arbitraire culturel» et des rapports de force. Il aurait confirmé par sa sociologie les thèses philosophiques de Nietzsche sur les valeurs.

Faut-il accorder créance à ces interprétations hyperboliques des trois noyaux durs?

\section{Montaigne}

Marquons d'abord, pour ne plus y revenir, l'immense distance qui sépare Montaigne et Geertz. Geertz et les «culturalistes » font de la socialisation l'alpha et l'oméga de l'explication du comportement. Pour Montaigne, les opinions sur toutes sortes de sujets varient d'une «nation» à l'autre. C'est vrai. Mais elles peuvent aussi se révéler très volatiles. En d'autres termes, la socialisation n'a nullement pour Montaigne les effets déterminants que lui prêtent les sociologues et les anthropologues «culturalistes». On le constate dans d'innombrables passages des Essais, comme dans ce passage cocasse où il montre que les croyances philosophico-religieuses elles-mêmes, loin de représenter un effet indélébile de la socialisation, peuvent au contraire se révéler très fragiles: un Grec membre de la secte stoïcienne, raconte-t-il, «lâcha un peu indiscrètement un pet en disputant, en présence de son école, et se tenait en sa maison, caché de honte [...]»: la dite honte est bien un effet de la socialisation. Mais cet effet devait se dissiper tout d'un coup lorsqu'un de ses amis «ajoutant à ses consolations et raisons l'exemple de sa liberté, se mettant à péter à l'envi avec lui, il lui ôta ce scrupule, et de plus le retira à sa secte Stoïque» (Essais, op. cit., p. 292).

L'argument «relativiste» de Montaigne prend chez Geertz et les autres une forme radicale parce qu'ils l'associent à une utilisation abusive du principe du tiers exclu: ou les règles de comportement sont objectivement fondées ou elles ne le sont pas. C'est méconnaître la possibilité que certaines règles soient conventionnelles, celles qu'on qualifie de «coutumes», et que d'autres ne le soient pas: celles qui sont fondées sur des raisons. C'est méconnaître aussi qu'une règle peut être l'expression symbolique d'une valeur fondée sur des raisons fortes, la règle relevant, elle, de la convention. Anglais et Français valorisent la politesse, mais l'expriment différemment. En tout cas, la perspective de Geertz a, entre autres inconvénients, celui de gommer la distinction qu'on repère dans toutes les langues entre les «coutumes» et les normes et valeurs auxquelles est normalement refusé le statut de coutumes.

Revenons à la question de l'excision, dont on peut noter en passant qu'elle n'a pas échappé à l'attention de Montaigne (Essais, op. cit., p. 282). Shweder (2000) paraît admettre, je l'ai dit, que le sentiment d'indignation qu'éprouve couramment l'observateur occidental à l'évocation de la pratique de l'excision est d'origine culturelle. C'est sous l'effet de la «fausse conscience» qu'il aurait l'impression que sa réaction a un fondement, non pas culturel, mais rationnel. 
N'est-il pas plus simple d'admettre qu'il n'y a là aucune fausse conscience, mais que l'on a bel et bien des raisons de condamner ladite pratique. On comprend que toute société ait tendance à mettre en place des rites destinés à faciliter la formation de l'identité personnelle et l'intégration sociale, et que l'excision ait une fonction de ce genre. On comprend très bien que la Sierra-Leonaise de Shweder ait tenu à passer dans son pays pour une vraie Sierra-Leonaise. Mais on sait aussi que la construction de l'identité personnelle et l'intégration sociale peuvent passer par d'autres voies et qu'en tout cas, dès lors qu'elles peuvent être favorisées par d'autres moyens que par le recours à la cruauté, ces autres voies tendent à être préférées.

Lorsque l'observateur occidental apprend que l'on coupe la main des voleurs dans certaines sociétés, il a couramment une réaction d'indignation, voire de répulsion. Comme dans le cas de l'excision, on peut en principe voir dans cette réaction une émanation de la culture occidentale. En fait, aucun anthropologue n'a osé, à ma connaissance, soutenir une telle thèse. Car, comme tout un chacun, l'anthropologue le plus «culturaliste» admettrait que la réaction en question est fondée sur des raisons faciles à énoncer, à savoir que, s'il est vrai que toute société doit mettre en place des mécanismes de contrôle social, il est également vrai que, lorsqu'on a trouvé le moyen de remplacer un mécanisme attentatoire à l'intégrité et à la dignité du coupable par un mécanisme tout aussi efficace mais dénué de barbarie, on incline à préférer le second. En reconnaissant que la réaction de l'observateur est l'effet d'une théorie implicite, de raisons, on se dispense de la mettre sur le compte de la «fausse conscience».

Cette théorie implicite dont je suppose qu'elle inspire plus ou moins consciemment la réaction de l'observateur occidental n'est autre que celle que Durkheim développe explicitement dans La Division du travail: quand on analyse l'histoire des sociétés, on observe une tendance de longue durée à l'invention de procédures permettant de donner des formes plus douces au contrôle social, nous dit-il. Cette tendance est une manifestation d'une valeur pérenne et fondamentale, qui inspire la vie morale et politique de toute société: l'individualisme. L' «individualisme» au sens moral, i.e. la demande par l'individu du respect de sa personne en tant que personne, est, nous dit Durkheim, «de tout temps », bien que son expression puisse être plus ou moins encouragée par les circonstances et par les évolutions structurelles, comme la tendance à l'approfondissement de la division du travail (Boudon, 2002). C'est pourquoi on constate que le droit pénal tend à diminuer en importance au profit du droit civil. C'est pourquoi les rituels visant à favoriser la construction de l'identité personnelle et l'intégration sociale prennent des formes de plus en plus douces, de plus en plus respectueuses de l'individu: de son intégrité physique; de la dignité de sa personne.

A la suite de Durkheim, on peut donc supposer que c'est pour des raisons fortes et non par application de règles qu'il aurait machinalement intériorisées au cours de la socialisation que l'observateur occidental juge que l'excision est un acte barbare et qu'il considère comme légitime que le législateur en interdise la pratique à toute personne, quelle que soit son origine culturelle. Un avantage subsidiaire de cette interprétation est qu'on peut alors prendre la réaction de l'observateur occidental à sa valeur faciale. On évite ainsi de lui imputer une «fausse conscience» au statut problématique; et l'on exonère l'anthropologue et 
le sociologue d'une mission impossible: expliquer les raisons pour lesquelles il est en droit d'affirmer que son voisin, que l'excision indigne, est atteint de fausse conscience, alors que lui-même aurait le privilège de la clairvoyance.

Bref, il est impossible d'assimiler toute croyance collective normative à une convention ou à une coutume. Il est vrai que les mathématiques sont un produit culturel que nous devons à la Grèce et à l'Egypte, mais d'un autre côté $2+2=4$ n'est pas une vérité culturelle. Ce qui est vrai des vérités mathématiques l'est aussi de certaines règles normatives. Toutes les langues font une différence entre coutumes et valeurs: entre les règles conventionnelles et celles qui ne le sont pas. Qu'est-ce qui permet d'affirmer que ces distinctions linguistiques sont dépourvues de fondement?

La question soulevée par Geertz et par les culturalistes pose comme une évidence que l'on peut trancher entre deux positions contradictoires, celle qui voit les croyances normatives comme conventionnelles et celle qui les voit comme rationnelles. En raison du cadre binaire dans lequel elle est formulée, elle apparaît finalement comme dénuée de sens.

\section{Hume}

L'énoncé correct du théorème de Hume est le suivant: on ne peut tirer une conclusion à l'impératif de prémisses toutes à l'indicatif. Ou: en général une conclusion prescriptive résulte d'un système de raisons dont certaines peuvent être à l'indicatif, mais dont une au moins doit être à l'impératif. Il suffit en effet que, dans un système de raisons, une seule raison soit à l'impératif pour qu'on puisse tirer dudit système une conclusion à l'impératif. Un raisonnement aussi simple que «les feux rouges sont une bonne chose, car sans eux la circulation serait encore pire» suffit à montrer qu'un jugement normatif dérive couramment à la fois de raisons factuelles et de raisons de caractère normatif.

Tout le monde ne croit pas à l'existence d'un gouffre entre le factuel et le normatif. Pour me limiter à un seul exemple, la sociologie fonctionnaliste (dans ses variantes scientifiquement efficaces) non seulement n'y croit pas, mais elle suffit à réfuter le corollaire qu'on tire à tort du théorème de Hume. Elle se donne pour programme de montrer que les gens valorisent positivement ou négativement une institution ou un état de choses dès lors que l'institution ou l'état de choses en question entraînent des conséquences qu'ils perçoivent comme positives ou négatives sur le fonctionnement de tel système social. Ainsi, la sociologie fonctionnaliste des inégalités a montré que l'opinion publique accepte facilement les inégalités dès lors qu'elle les voit comme fonctionnelles. Ici, les attitudes des acteurs sociaux sont expliquées par les raisons qu'ils ont de les adopter, ces raisons ayant trait aux conséquences factuelles que tel ou tel degré d'inégalités a des chances d'engendrer: une explication dont on ne voit pas pourquoi elle ne pourrait être tenue pour parfaitement acceptable.

De même, on peut expliquer les croyances collectives en matière d'organisation de la cité par le fait que telle institution est perçue comme satisfaisant ou non tel critère, par exemple: le respect de la dignité du citoyen.

Le corollaire qu'on tire couramment du théorème de Hume est donc non seulement un paralogisme; de plus, il contredit l'observation. Mais la popularité de l'idée reçue qu'il a engendrée contribue à expliquer qu'on traite comme une 
évidence l'idée selon laquelle les croyances normatives seraient nécessairement conventionnelles.

\section{Max Weber}

Max Weber est souvent présenté par ses commentateurs comme un hyper-relativiste: il aurait affirmé que les sociétés sont animées par des conflits endémiques et insolubles entre systèmes concurrents de valeurs. Bryan Turner (1992) naguère, Leo Strauss (1953) hier ont offert des exemples particulièrement représentatifs des interprétations hyperboliques qui ont été proposées du «noyau dur» emprunté à Weber.

En réalité, on peut résumer l'argumentation elliptique de sa conférence sur $L e$ Savant et le politique de la façon suivante: la théorie la plus solide est toujours fondée sur des principes. Or, qui dit principes dit propositions premières et par conséquent, propositions non fondées. Ainsi, toute théorie, y compris la théorie scientifique la plus solide, repose sur des propositions non fondées. Weber ne fait que reconnaître par-là une évidence logique: c'est pourquoi sans doute il ne s'y appesantit pas. Simmel reconnaît la même évidence lorsqu'il avance qu'on ne peut discuter une chaîne argumentative qu'à partir de son second maillon. Le célèbre «trilemme de Münchhausen» de Hans Albert réexprime la même idée.

Mais cette évidence logique contredit un a priori répandu, à savoir qu'une théorie ne saurait être solide si ses fondements ne le sont pas. De cet a priori, on a déduit que toute théorie solide devait être fondée soit sur des évidences, soit sur des principes garantis par une autorité absolue, la Nature et Dieu étant les autorités les plus souvent invoquées. Leo Strauss opte pour la Nature: il est un partisan de la théorie du droit naturel. Bryan Turner, lui, est également convaincu qu'une théorie solide ne peut s'appuyer que sur des principes solides. Mais, comme il ne croit ni à la Nature ni à la Révélation, il en conclut qu'il n'existe pas de théorie solide.

C'est donc de leurs propres prénotions que Leo Strauss ou Bryan Turner tirent l'idée que Weber ne reconnaît pas l'existence de vérités. Elles seraient «construites». Il n'y aurait pour Weber, selon ces auteurs, aucune vérité: ni morale, ni scientifique, ni politique. Leo Strauss en conclut que Weber est le père du relativisme moderne. Les valeurs seraient selon lui l'effet du libre choix : il ne serait pas plus noble de produire une grande découverte scientifique que de collectionner des timbres-poste. Voegelin (1952) et Allan Bloom (1987) vont encore plus loin: Weber serait largement responsable de la décomposition morale des sociétés modernes. Bryan Turner, à partir des mêmes arguments, fait aujourd'hui de Weber le premier des postmodernistes et le félicite, là où Leo Strauss, hier, le blâmait pour les mêmes motifs.

En fait, Weber ne tire nullement de l'idée que les valeurs reposent en dernier ressort sur des principes non fondés la conclusion qu'elles relèveraient de l' «arbitraire culturel», de la «convention». Il n'adhère pas non plus à l'idée selon laquelle les valeurs s'imposeraient sous l'effet de rapports de force. Ce qu'il veut dire, c'est que les théories normatives, comme les théories scientifiques, se développent sur la base de principes non démontrés, mais qui donnent naissance à des programmes qui, soit se consolident progressivement, soit sont abandonnés s'ils se révèlent non viables. Il y a chez Weber un évolutionnisme qu'on peut qualifier 
de «bien tempéré». La sélection des idées normatives, des idées politiques, voire des idées religieuses ou philosophiques obéit, comme celle des idées scientifiques, à un processus qu'il qualifie de «rationalisation diffuse » (Durchrationalisierung). Sous l'effet de ce processus, des principes non démontrables se trouvent consolidés a posteriori par l'intérêt des conséquences sur lesquelles ils débouchent.

Au total, Weber esquisse une théorie de la connaissance et de l'action que je qualifierais volontiers de programmatiste.

Ainsi, lorsqu'une institution politique paraît devoir garantir plus efficacement la dignité des individus, elle tend à être perçue comme légitime, à faire l'objet d'une demande collective et, si les circonstances s'y prêtent, à s'inscrire dans les faits. C'est le cas du droit de grève, par exemple, ou de la reconnaissance de syndicats indépendants des autorités de l'entreprise et des autorités politiques. Si ces institutions se sont imposées, c'est qu'elles représentent un moyen efficace de protection des ouvriers ou des employés. La nécessité de protéger ces catégories dérive du principe du respect de la dignité de tout sociétaire. Ce principe n'est pas démontrable. Mais il inspire la vie politique et lui donne une orientation. Avant que ne s'installe le droit de grève, on pouvait avoir l'impression que régnait sur ce sujet un «polythéisme des valeurs» ou une «guerre des dieux»: d'un côté, les valeurs et les intérêts des employeurs; de l'autre, les valeurs et les intérêts des ouvriers et des employés. Aujourd'hui, plus personne ne conteste le droit de grève. Tout au plus propose-t-on de l'«aménager».

On sait qu'une organisation politique obéissant au principe de la distribution des pouvoirs (Montesquieu) est préférable à un pouvoir concentré. Cette vérité paraissait si évidente à Hume (1972 [1741], p. 187) qu'elle lui semblait devoir s'imposer rapidement. Elle ne s'est pas imposée rapidement, loin de là. Elle rencontre toujours des résistances. Mais, quelles que soient les difficultés de sa mise en œuvre, le principe de la séparation des pouvoirs s'est progressivement imposé de manière irréversible à partir du moment où l'on eut constaté que son application entraînait effectivement une affirmation du droit des personnes et un approfondissement de la paix civile. Il est même question que le Parti communiste chinois débatte dans son prochain congrès de l'intérêt qu'il y aurait à reconnaître en son sein l'existence de trois pouvoirs indépendants l'un de l'autre: décisionnaire, exécutif et disciplinaire (Courrier international, $\mathrm{n}^{\circ}$ 612, 25-31 juillet 2002, p. 16). L'affrontement, la "guerre des dieux», caractérise l'installation des valeurs dans le court et le moyen terme. Dans le long terme, celle-ci apparaît comme le résultat d'un processus de sélection rationnel.

Des considérations identiques pourraient être développées à propos de toutes sortes d'autres principes: pensons à la séparation de l'Eglise et de l'Etat, à la liberté d'opinion, aux garanties apportées à la liberté d'entreprendre, au droit à l'éducation, à l'égalité des femmes, à la confirmation légale des droits de propriété, à la notion de Rechtsstaat (Etat de droit), au droit d'ingérence, au principe de subsidiarité, aux droits de propriété, etc. Tous ces principes se sont installés dans un contexte de «guerre des dieux». Ils font désormais l'objet d'un consensus. Ils ne donnent jamais lieu dans les sociétés occidentales modernes à des objections directes.

C'est lorsqu'un principe n'est pas encore installé qu'il évoque les images du «polythéisme des valeurs» et de la «guerre des dieux». Rappelons-nous les 
débats d'hier entre protectionnistes et antiprotectionnistes. On sait aujourd'hui que le protectionnisme est en général un principe pervers, car il inspire des mesures qui permettent de régler des problèmes présents, mais en obérant lourdement l'avenir. Il a été l'objet de débats qui évoquèrent aux yeux des contemporains l'image d'une «guerre des dieux », alors que ces débats nous apparaissent $a$ posteriori comme la dramatisation politique de la concurrence entre une idée traditionnelle et une idée nouvelle.

En se déplaçant par la pensée dans le futur, on imagine facilement que ce qui apparaît aujourd'hui comme une «lutte inexpiable» entre les valeurs incompatibles de la souveraineté intégrale et de la souveraineté limitée apparaîtra demain comme la manifestation de la concurrence entre un principe traditionnel sommaire (plus un gouvernement a de latitude d'action, plus il a la capacité de satisfaire le bien commun ou de répondre aux demandes du public) et un principe sans doute plus complexe à définir et plus difficile à mettre en œuvre, mais aussi plus réaliste et par suite plus efficace. Les abandons de souveraineté auxquels ont consenti les pays de la communauté européenne leur ont valu une capacité accrue de résistance aux chocs venus de l'extérieur. Si la France en était restée au franc lors des élections présidentielles de 2002 qui placèrent le dirigeant du Front National en position de concourir pour le second tour, il est bien possible que la monnaie nationale ait eu à en souffrir. Les processus de sélection rationnelle diffuse des idées feront que les idées des «souverainistes » apparaîtront sans doute demain comme ayant un intérêt de caractère exclusivement historique.

De même, l'idée de la gratuité des services publics est en France si ancrée que souligner qu'elle engendre des effets pervers relève du sacrilège. Sur ce point, on n'a même pas encore atteint en France la phase de la «guerre des dieux ». Demain, on acceptera sans doute comme allant de soi l'idée que, comme l'indique Padioleau (2002), «des demandes sans garde-fou et des droits sans limites consument des valeurs tenues pour essentielles du service public (égalité, gratuité, etc.) par suite des effets d'encombrements, de surcharge et de coûts inflationnistes ».

A l'instar du phlogistique, le protectionnisme, le principe de la souveraineté intégrale ou le principe de la gratuité intégrale des services publics ont été ou seront relégués dans les livres d'histoire des idées par les processus de sélection rationnelle des idées.

Rien n'interdit évidemment que les intérêts ne puissent temporairement prévaloir sur les principes les mieux installés. Mais ce qu'il importe de noter, c'est que ces épisodes ne remettent pas en cause les principes: les dispositions protectionnistes prises par le président américain George W. Bush en matière d'agriculture et d'acier ont déconcerté; elles ont été justifiées par des raisons de caractère conjoncturel; elles ne se sont nullement accompagnées d'une remise en cause du principe selon lequel il est préférable d'éviter les mesures protectionnistes toujours génératrices d'effets pervers à long terme.

Ces exemples me paraissent illustrer les processus que Weber avait en l'esprit lorsqu'il parle de «rationalisation diffuse» ou de «polythéisme des valeurs». Bref, Weber lui-même n'a jamais tiré du fait que toute théorie repose sur des principes, par définition même invérifiables, la conséquence qu'aucune théorie normative ne peut être considérée comme solide et comme fondée et que doive s'installer définitivement sur tout sujet le règne de la «guerre des dieux » et du «polythéisme des valeurs». 
On ne tire cette conclusion que si l'on accepte la lecture relativiste souvent proposée de Weber, laquelle relève du contresens, et que si, de surcroît, on se donne une hypothèse fondée sur une utilisation abusive du principe du tiers exclu: ou bien les normes et les valeurs sont fondées sur des raisons solides ou bien elles ne le sont pas et doivent dans ce cas être mises sur le compte de l'arbitraire culturel, de la «convention» ou de la lutte des intérêts.

\section{FIN DU RELATIVISME CULTUREL?}

Pour conclure sur le relativisme culturel: il hyperbolise des noyaux durs à l'aide de questions binaires reposant sur une utilisation abusive du principe du tiers exclu.

Comme dans le cas du relativisme cognitif, il faut aussi tenir compte de l' «utilité » au sens de Pareto des théories affirmant le relativisme culturel: plaçant toutes les sociétés sur un pied d'égalité, elles préservent leur dignité. Si le relativisme culturel est fondé, il est impossible d'affirmer qu'une société est plus avancée qu'une autre, qu'elle a des institutions supérieures à une autre. Le règne de la «bienveillance universelle» peut alors s'instaurer: on est invité à considérer toutes les cultures comme également dignes de respect. Bref, la congruence entre le relativisme culturel et les exigences de l'égalitarisme explique certainement l'influence et la large diffusion du premier.

Mais, par application du théorème tiré des Formes élémentaires de Durkheim, selon lequel une croyance collective non fondée ne saurait se maintenir sur le long terme, la fragilité du relativisme culturel, son caractère d'hyperbole greffée sur des noyaux durs devrait être de mieux en mieux perçu. Shweder (2000) tente de nous convaincre que l'excision ne peut être jugée de l'extérieur, en conformité avec sa thèse de l'incommensurabilité des cultures. Mais le «culturalisme » atteint ici ses limites. On attend encore le «culturaliste» qui nous expliquerait qu'on ne peut juger de l'extérieur la lapidation des femmes jugées coupables d'adultère et les diverses mutilations que le droit pénal inflige aux coupables dans certaines sociétés.

Université de Paris-Sorbonne (Paris-IV)

Institut de France (Académie des sciences morales et politiques)

\section{RÉFÉRENCES BIBLIOGRAPHIQUES}

Aya, R. (2001), The Curse of Cognitive Cultural Relativism, in F. Lindo and Mies van Niekerk, Dedication and Detachment, Essays in Honour of Hans Vermeulen, Amsterdam, Aksant, 33-41.

Bloom, A. (1987), The Closing of the American Mind, New York, Simon \& Schuster, 1987.

Boltanski, L., Thévenot, L. (1991), De la justification: Les économies de la grandeur, Paris, Gallimard.

Boudon, R. (2002), Déclin de la morale? Déclin des valeurs? Paris, PUF et Québec, Nota Bene.

Bunge, M. (1999), The Sociology-Philosophy Connection, Londres / New Brunswick (USA), Transaction. 
Douglas, M., Ney, S. (1998), Missing Persons: a Critique of Personehood in the Social Sciences, Londres, Sage.

Durkheim, E. (1979 [1910]), Les Formes élémentaires de la vie religieuse, Paris, Presses Universitaires de France.

Feyerabend, P. (1975), Against Method, Londres, N.L.B., 1975; en fr. : Contre la méthode, Paris, Seuil, 1979.

Geertz, C. (1984), «Distinguished Lecture: Anti anti-relativism», American anthropologist, vol. 86, $\mathrm{n}^{\circ} 2,263-278$.

Goldhagen, D. J. (1997), Hitler's Willing Executioners; Ordinary Germans and the Holocaust, New York, A. Knopf.

Granet, M. (1990), Etudes sociologiques sur la Chine, Paris, PUF.

Henrich, J., Boyd, R., Bowles, S., Camerer, C., Fehr, E., Gintis, H., McElreath, R. (2001), «In Search of Homo Economicus: Behavioral Experiments in 15 Small-scale Societies », American Economic Review, vol. 91, n 2, May, 73-78.

Hume, D. (1972 [1741]), Essais politiques, Vrin, Paris. Tr. de Essays Moral and Political, London, printed for A. Millar, $3^{\mathrm{e}}$ ed. 1748.

Huntington, S. (1996), The Clash of Civilizations and the Remaking of the World Order, New York, Shuster and Shuster. Tr. fr. : Le Choc des civilisations, Paris, O. Jacob, 1997.

Kuhn, T. (1962), The Structure of Scientific Revolutions, Chicago, U. of Chicago Press.

Lévy-Bruhl, L. (1960 [1922]), La Mentalité primitive, Paris, PUF.

Needham, R. (1972), Belief, Language and Experience, Oxford, Blackwell, 1972.

Padioleau, J. (2002), «Une piété française: 'La réforme de 1'Etat'», Le Débat, n 119, Mars-Avril, 2034.

Shweder, R. A. (1991), Thinking through Cultures, Expeditions in Cultural anthropology, Cambridge, Harvard University Press. (2000), What about «female genital mutilation» and why understanding culture matters in the first place, Daedalus, 129, 4, 209-232.

Sokal, A. et Bricmont, J. (1997), Impostures intellectuelles, Paris, O. Jacob.

Strauss, L. (1953), Natural Right and History, Chicago, Chicago U. Press.

Turner, B. S. (1992), Max Weber : from History to Modernity, London, Routledge.

Voegelin, E. (1952), Toward a New Science of Politics, Chicago, IL., The University of Chicago Press.

Whorf, B. L. (1969), Linguistique et anthropologie. Les origines de la sémiologie, Paris, Denoël. 